\title{
THE REGULARITY OF MAPPINGS WITH A CONVEX POTENTIAL
}

\section{LUIS A. CAFFARELLI}

In this work, we apply the techniques developed in [C1] to the problem of mappings with a convex potential between domains.

That is, given two bounded domains $\Omega_{1}, \Omega_{2}$ of $R^{n}$ and two nonnegative real functions $f_{i}$ defined in $\Omega_{i}$ that are bounded away from zero and infinity, we want to study the map $\vec{v}=\nabla \psi$ for a Lipschitz convex $\psi$, such that $\nabla \psi$ maps $\Omega_{1}$ onto $\Omega_{2}$ in the a.e. sense and in some (weak) sense.

$$
f_{2}(\nabla \psi) \operatorname{det} D_{i j} \psi=f_{1}(X) \text {. }
$$

In recent work $Y$. Brenier showed existence and uniqueness of such a map (provided that $\left|\partial \Omega_{i}\right|=0$ ) under the obvious compatibility condition

$$
\int_{\Omega_{1}} f_{1}=\int_{\Omega_{2}} f_{2} .
$$

The map $\nabla \psi$ is into $\Omega_{2}$ in the sense that $\psi=\sup L_{\alpha}$ with $L_{\alpha}$ linear functions and $\nabla L_{\alpha} \in \Omega_{2}$. The Monge-Ampère equation (1) is satisfied in the weak sense.

$$
\int_{\Omega_{2}} \eta(Y) f_{2}(Y) d y=\int_{\Omega_{1}} \eta(\nabla \psi) f_{1}(X) d X
$$

for any continuous function $\eta$, (here $\nabla \psi$ is understood in the $L^{\infty}$-sense).

As can be seen by an elementary example, this definition is strictly weaker than the classical definition of weak solution by Pogorelov, since it is unable to see the singular part of $\operatorname{det} D_{i j} \psi$.

On the other hand, if both $\Omega_{i}$ are convex, Pogorelov [P] constructed a weak solution in the Alexandrov sense.

The purpose of this note is to show

Theorem. If $\Omega_{2}$ is convex and $f_{i}, 1 / f_{i}$ are bounded, then Brenier's solution is a weak solution in the sense of Alexandrov.

Further, $\psi$ is strictly convex and $C^{1, \beta}$ for some $\beta$.

If $f_{i}$ are continuous, $\psi \in W_{\mathrm{loc}}^{2, p}$ for every $p$.

If $f_{i}$ are $C^{\bar{a}}, \psi \in C^{2, a}$ for any $0<a<\bar{a}$.

Further regularity follows from classical standard estimates.

The main difficulty in the proof of the theorem consists in showing that Brenier's solution is indeed a solution in the sense of Alexandrov, i.e., that

Received by the editors September 20, 1990.

1991 Mathematics Subject Classification. Primary 35J65, 35B65.

Supported in part by N.S.F. grants DMS 8804567 and INT 8902934. 
$\operatorname{det} D_{i j} \psi$ has no singular part and that $\psi$ is strictly convex (in the sense of $[\mathrm{Cl}])$.

After this, the results in $[\mathrm{C} 1, \mathrm{C} 2, \mathrm{C} 3]$ provide the complete regularity theory.

To see that the condition needed on $\Omega_{2}$ is of geometrical nature, and neither a topological or differentiability one, consider the following examples.

(i) Let, on $R^{2}$,

$$
\psi_{0}=\left|x_{1}\right|+\frac{1}{2}\left(x_{1}^{2}+x_{2}^{2}\right) .
$$

Then $\nabla \psi_{0}=\left(x_{1}+\operatorname{sign} x_{1}, x_{2}\right)$ is the (unique) map from the unit disc onto the two shifted halfdiscs, $D_{1}^{+}+(1,0)$ and $D_{1}^{-}-(1,0)$. In the Alexandrov sense, though, $\operatorname{det} D_{i j} \psi$ has a singular measure along $\left\{x_{1}=0\right\}$ and the image $\nabla \psi$, as a multivalued map, fills the strip $\left|x_{1}\right| \leq 1$.

(ii) Bridge now the two shifted halfdiscs of the previous example with an $\varepsilon$ horizontal strip $\left\{\left|x_{2}\right|<\varepsilon,\left|x_{1}\right| \leq 1\right\}$, make an $\varepsilon$-smoothing of the new domain to make it $C^{\infty}$ with total area

$$
\left|\Omega_{2}\right|=\Pi=\left|D_{1}\right|
$$

and consider the Brenier map $\nabla \psi_{\varepsilon}: D_{1} \rightarrow \Omega_{\varepsilon}$ (with $f \equiv 1$ ). It follows from Brenier's theorem that $\psi_{\varepsilon}$ converges uniformly to $\psi_{0}$, and hence in the sense of Alexandrov that

$$
\lim \left|\operatorname{det} D_{i j} \psi_{\varepsilon}\left(D_{1}\right)\right| \geq\left|\operatorname{det} D_{i j} \psi_{0}\left(D_{1}\right)\right|
$$

(| | denotes total mass).

But since $\left|\operatorname{det} D_{i j} \psi_{0}\left(D_{1}\right)\right|=\left|D_{1}\right|+\left|\left\{\left|x_{1}\right|<1\right\}\right|$ (the added strip due to the singular part of $\left.\operatorname{det} D_{i j} \psi_{0}\right)$, it follows that $\operatorname{det} D_{i j} \psi_{\varepsilon}$ must also have a singular part for $\varepsilon$ small enough.

The difficulty posed by this counterexample is easily solved by the following remark.

Lemma 1. If $\psi$ is locally Lipschitz and convex then

(a) a point of Lebesgue differentiability of $\nabla \psi$ (as an $L^{\infty}$ function) is a point of continuity for $\nabla \psi$;

(b) if $\nabla \psi$, in the a.e. sense, is contained in $\Omega$, then, in the multivalued map sense, $\nabla \psi$ is contained in $\Gamma(\Omega)$, the closed convex envelope of $\Omega$.

Proof. (a) If 0 is not a point of continuity for $\nabla \psi$ we have at zero at least two supporting planes that can be normalized to be $Z= \pm a x_{1} \quad(a>0)$ by subtracting an appropriate linear function.

Let $\Gamma_{\varepsilon}^{+}$be the cone

$$
\Gamma_{\varepsilon}^{+}=\left\{X:\left\langle X, e_{1}\right\rangle \geq(1-\varepsilon)|X|\right\} .
$$

Along this cone a supporting plane at a point $X_{0}=\left(x^{0}, y^{0}\right),\left(x^{0}\right.$ a scalar $)$,

$$
Z=\langle\vec{v}, X\rangle+a
$$

must satisfy

$$
Z(0)=a<0 \text { and } Z\left(X_{0}\right)=v_{1} x^{0}+\left\langle\vec{v}_{2}, y^{0}\right\rangle+a \geq a x^{0} .
$$


In particular,

$$
v_{1} x^{0}+\left\langle\vec{v}_{2}, y^{0}\right\rangle \geq a x^{0} \quad \text { or } \quad v_{1} \geq a-\frac{1}{x^{0}}\left\langle\vec{v}_{2}, y_{0}\right\rangle \text {. }
$$

In particular, since along $\Gamma,\left|y^{0}\right|<C \varepsilon x^{0}$ and $\left|\vec{v}_{2}\right| \leq C$ because $\psi$ is Lipschitz, $v_{1}+C \varepsilon \geq a$.

For $\varepsilon$ small enough, $v_{1} \geq a / 2$. Conversely in the opposite cone, $\Gamma_{\varepsilon}^{-}, v_{1} \leq$ $-a / 2$ and 0 is not a point of Lebesgue differentiability.

This proves part (a).

In order to prove part (b), since values of Lebesque differentiability of $\nabla \psi$ are, after (a), mapped in $\Omega$, we need to prove the following:

Let 0 be a point of discontinuity for $\nabla \psi$ and $\Sigma$ the convex, (nontrivial) set of supporting planes to $\psi$ at 0 .

We will say that $\vec{u}$ is a strongly extremal point of $\Sigma$ if $\Sigma$ is tangent from inside to some sphere at $\vec{u}$.

For bounded convex sets, strongly extremal points are dense in the set of extremal points, and therefore, it is enough to show that strongly extremal points, $\vec{u}$, are limits of points of continuity for $\nabla \psi$.

By subtracting a linear function and a rotation we may assume that $\vec{u}=0$ and $\Sigma \subset B_{R}\left(R e_{1}\right)$ (the ball of radius $R$ and center $R e_{1}$. )

We then show that along any cuspidal domain around the ray $-\lambda e_{1}$, all possible supporting vectors converge to zero.

Indeed, any sequence $v^{(k)}$ must converge to a point in $\Sigma$, and hence their first component satisfy

$$
\underline{\lim } v_{1}^{(k)} \geq 0 \text {. }
$$

On the other, $\vec{u}=0$ being a supporting plane, $\psi \geq 0$ and from the argument in part (a).

$$
\underline{\lim }\left\langle\nabla \psi,-e_{1}\right\rangle \geq-C \varepsilon
$$

along any $\Gamma_{\varepsilon}$ cone around the $-e_{1}$ axis.

Hence $\lim v_{1}^{k}=0$ and $v^{k}$ must converge to zero along any cuspidal domain.

We now study solutions $\psi$ when $\Omega_{2}$ is already convex.

More precisely

Lemma 2. Consider a convex function $\varphi$ such that

(a) $\varphi=\sup L_{\alpha}, L_{\alpha}$ linear, $\nabla L_{\alpha} \in \Omega_{2}$;

(b) $\left|\Gamma\left(\Omega_{2}\right) \backslash \Omega_{2}\right|=0$ (| | Lebesgue measure, $\Gamma\left(\Omega_{2}\right)$ the convex envelope of $\left.\Omega_{2}\right)$;

(c) for any continuous $\eta$

$$
C_{1} \int \eta(\nabla \varphi)(X) d X \leq \int_{\Omega_{2}} \eta(Y) d Y \leq C_{2} \int_{\Omega_{1}} \eta(\nabla \varphi(X)) d X
$$

$\left(\nabla \varphi\right.$ as an $L^{\infty}$ function $)$

Then, in the sense of Alexandrov $\varphi$ satisfies (in all of $R^{n}$ )

$$
C_{1} \chi_{\Omega_{1}} \leq \operatorname{det} D_{i j} \varphi \leq C_{2} \chi_{\Omega_{1}}
$$


Proof. It is clear that

$$
C_{1} \chi_{\Omega_{1}} \leq \operatorname{det} D_{i j} \varphi
$$

Indeed, for any compact set $K_{1} \subset \Omega_{1}, K_{2}=\nabla \varphi\left(K_{1}\right)$ in the multivalued sense is compact. If $\eta$ is any continuous majorant of $\chi_{K_{2}}$

$$
\int \eta(Y) d x \geq C_{1} \int \eta(\nabla \varphi) d X \geq C_{1}\left|K_{1}\right|
$$

In particular if $\nabla \varphi(S)$ for $S \subset \Omega_{1}$ has measure zero, also $S$ must have measure zero.

To prove the second part of the inequality, we recall that given $K$ compact in the $X$ space, the set $D$, in the $\nabla$ space of those $\vec{u}$ such that

$$
(\nabla \varphi)^{-1}(\vec{u}) \cap K \neq \varnothing \text { and }(\nabla \varphi)^{-1}(\vec{u}) \cap \mathscr{C} K \neq \varnothing
$$

has measure zero. (See [C1], the proof of the remark in p. 137.)

Hence $\left|(\nabla \varphi)^{-1}\left(\nabla \varphi\left(K_{1}\right)\right) \backslash K_{1}\right|=0$.

But now for any $K_{1}$ and any continuous majorant $\eta$ of $\nabla \varphi\left(K_{1}\right)$, we have

$$
\left|\nabla \varphi\left(K_{1}\right)\right| \leq \int_{\Gamma\left(\Omega_{2}\right)} \eta(Y) d Y=\int_{\Omega_{2}} \eta(Y) d Y \leq C_{2} \int_{\Omega_{2}} \eta(\nabla \varphi) d X .
$$

If now $0 \leq \eta \leq 1$ and $\eta$ converges uniformly to zero on compact sets outside $\nabla \varphi\left(K_{1}\right), \eta(\nabla \varphi)$ converges uniformly to zero on compact subsets of $\Omega_{1} \backslash$ $(\nabla \varphi)^{-1}\left(\nabla \varphi\left(K_{1}\right)\right)$.

Therefore $\int \eta(\nabla \varphi(X)) d X$ is controlled by $\left|\chi_{\nabla \varphi^{-1}\left(\nabla \varphi\left(K_{1}\right)\right.}\right|=\left|\chi_{K_{1}}\right|$ and the proof is complete.

At this point we have been able to show that $\operatorname{det} D_{i j} \varphi$ has no singular measure.

To complete our argument; let us show that $\left.\varphi\right|_{\Omega_{1}}$ is strictly convex (in the sense of [C1], i.e., that every tangent plane has only one contact point with graph of $\varphi$ ).

We first point out that if $\varphi$ is a globally Lipschitz convex nonnegative function and $\varphi \equiv 0$ on a line (say in the $e_{1}$ direction) then $\varphi_{e_{1}} \equiv 0$ and hence $\operatorname{det} D_{i j} \varphi \equiv 0$.

Therefore it is enough to prove.

Lemma 3. Let $\varphi$ be a globally Lipschitz convex nonnegative function, that satisfies, in the Alexandrov sense

$$
C_{1} \chi_{\Omega_{1}} \leq \operatorname{det} D_{i j} \varphi \leq C_{2} \chi_{\Omega_{1}} .
$$

Then, if the (convex) set $S=\{\varphi \equiv 0\}$ intersects $\Omega_{1}$ in more than a point, then $S$ has no extremal points (and contains therefore a line).

Proof. $\varphi$ being convex and globally Lipschitz, it has an asymptotic cone at infinity and $\nabla \varphi\left(R^{n}\right)$ is a bounded convex set with nonempty interior $\Gamma$.

We recall from [C1] that $S$ cannot have extremal points in $\Omega_{1}$, and since $\operatorname{det} D_{i j} \varphi \equiv 0$ on $\mathscr{C} \Omega_{1}$, it follows that $S$ can only have extremal points on $\partial \Omega_{1}$. 
As it was observed before Lemma $3, S$ cannot contain a line (if not $\operatorname{det} D_{i j} \varphi$ $\equiv 0$ ). Hence it must have a supporting plane, let us say $x_{1}=0$, that has only one contact point, say 0 , with $S$.

Then 0 must belong to $\partial \Omega_{1}$, and since $S$ has also a point in the interior of $\Omega_{1}$, we have the following geometric situation:

(i) $S \subset\left\{x_{1} \leq 0\right\}, S \cap\left\{x_{1}=0\right\}=0$, and hence $S \cap\left\{x_{1} \geq-M\right\}$ is compact for any $M$.

(ii) 0 also belongs to $\partial \Omega_{1}$ and hence $\Omega_{1} \subset B_{R_{0}}$ for some $R_{0}$.

(iii) $S$ has a point $X_{0}$ in (the interior of) $\Omega_{1}$.

In particular some ball $B_{\delta}\left(X_{0}\right) \subset \Omega_{1}$. As in [C1] we will study the renormalization of the functions $\varphi_{\varepsilon}=-\varepsilon\left(x_{1}+2 R_{0}\right)+\varphi$ on the set $\left\{\varphi_{\varepsilon}<0\right\}=S_{\varepsilon}$.

That is, $S_{\varepsilon}$ has nonempty interior since 0 and $X_{0} \in S_{\varepsilon}$ and, therefore, by an affine transformation we may obtain sets $S_{\varepsilon}^{*}$ and graphs $\varphi_{\varepsilon}^{*}$ such that $B_{1} \subset S_{\varepsilon}^{*} \subset B_{n}$.

$$
C_{1} \chi_{\Omega_{1}^{*}} \leq \operatorname{det} D_{i j} \varphi_{\varepsilon}^{*} \leq C_{2} \chi_{\Omega_{1}^{*}} \text { and } \varphi^{*} \equiv 0 \text { on } \partial D_{\varepsilon}^{*} .
$$

Since $S_{\varepsilon}$ converges uniformly to $S \cap\left\{x_{1}>-2 R_{0}\right\}$, a compact set, $S_{\varepsilon}$ is uniformly bounded for $\varepsilon$ small and therefore the affine transformation $T_{\varepsilon}$ that sends $S_{\varepsilon}$ in $S_{\varepsilon}^{*}$ is an expansion, i.e., $\left\|T_{\varepsilon}(X)-T_{\varepsilon}(Y)\right\| \geq C|X-Y|$, independently of $\varepsilon$.

In particular $\Omega_{1}^{*}=T_{\varepsilon}(\Omega) \supset T_{\varepsilon}\left(B_{\delta}\left(X_{0}\right)\right) \supset B_{C_{0} \delta}\left(X_{0}^{*}\right)$.

As in [C1] we now will get a contradiction by showing that both $\varphi_{\varepsilon}^{*}$ stays close to its infimum near $\partial S_{\varepsilon}^{*}$, and cannot do so.

On one hand, from Alexandrov estimate,

$$
\left|\varphi_{\varepsilon}^{*}(X)\right|^{n} \leq \operatorname{dist}\left(X, \partial S_{\varepsilon}^{*}\right) \text { Total mass }\left(\operatorname{det} D_{i j} \varphi_{\varepsilon}^{*}\right) \leq C d\left(X, \partial S_{\varepsilon}^{*}\right)
$$

since $\operatorname{det} D_{i j} \varphi^{*}$ is bounded.

On the other hand, consider $S_{\varepsilon, \delta / 2}^{*}=\left\{X \in S_{\varepsilon}^{*} \mid d\left(X, \partial S_{\varepsilon}^{*}\right) \geq C_{0} \delta / 2\right\}$.

There, $|\nabla \varphi| \leq \frac{C}{\delta}\left|\inf _{S_{\varepsilon}^{*}} \varphi\right|$. But

$$
\left|B_{C_{0} \delta}\left(X_{0}^{*}\right) \cap S_{\varepsilon, \delta / 2}^{*}\right| \sim C \delta^{n} .
$$

Hence

$$
\text { Volume }\left\{\nabla \varphi\left(S_{\varepsilon, \delta / 2}^{*}\right)\right\}=\int_{S_{\varepsilon, \delta / 2}^{*}} \operatorname{det} D_{i j} \varphi d X \geq C \delta^{n} .
$$

Hence $\sup _{S_{\varepsilon, \delta / 2}^{*}}|\nabla \varphi| \geq C \delta$ and

$$
\left|\inf _{S_{\varepsilon}^{*}} \varphi_{\varepsilon}^{*}\right| \geq C \delta^{2}
$$

Finally, we look at $T_{\varepsilon}(0)$.

On one side $T_{\varepsilon}(0)$ converges to $\partial S_{\varepsilon}^{*}$ when $\varepsilon$ goes to zero since the opposite supporting planes of $S_{\varepsilon},\left\{x_{1}=-A_{\varepsilon}\right\}\left\{x_{1}=B_{\varepsilon}\right\}$, remain uniformly separate (since they enclose $X_{0}$ and 0 ) and $B_{\varepsilon}$ tends to zero since $S_{\varepsilon}$ converges to $S$. 
On the other, the ratio

converges to one since

$$
\frac{\varphi_{\varepsilon}^{*}\left(0^{*}\right)}{\inf _{S_{\varepsilon}^{*}} \varphi_{\varepsilon}^{*}}=\frac{\varphi_{\varepsilon}(0)}{\inf _{S_{\varepsilon}} \varphi_{\varepsilon}}
$$

$$
\begin{gathered}
\left|\inf _{S_{\varepsilon}} \varphi_{\varepsilon}\right| \leq \sup _{x_{1} \in S_{\varepsilon}} \varepsilon\left(x_{1}+2 R_{0}\right), \\
\varphi_{\varepsilon}(0)=-2 \varepsilon R_{0}
\end{gathered}
$$

and $\sup _{x_{1} \in S_{\varepsilon}} x_{1}$ goes to zero when $S_{\varepsilon}$ converges to $S$.

The proof of the theorem is now complete.

\section{REFERENCES}

[B] Yann Brenier, Décomposition polaire et réarrangement monotone des champs de vecteurs, C. R. Acad. Sci. Paris Sér. I. Math. 305 (1987), 805-808.

[C1] Luis A. Caffarelli, A localization property of viscosity solutions to the Monge-Ampère equation and their strict convexity, Ann. of Math. (2) 131 (1990), 129-134.

[C2] - Interior $W^{2, p}$ estimates for solutions of the Monge-Ampère equation, Ann. of Math. (2) 131 (1990), 135-150.

[C3] __ Some regularity properties of solutions to Monge Ampère equation, Comm. Pure Appl. Math. (to appear).

[P] A. V. Pogorelov, Monge-Ampère equations of elliptic type, Noordhoff, Groningen, 1964.

Institute for Advanced Study, School of Mathematics, Princeton, New Jersey 08540 